\title{
Common and differential effects of docosahexaenoic acid and eicosapentaenoic acid on helper T-cell responses and associated pathways
}

\author{
Jaeho Lee ${ }^{1, \#}$, Yu Ri Choi, ${ }^{1, \#}$, Miso Kim ${ }^{1}$, Jung Mi Park ${ }^{2}$, Moonjong Kang ${ }^{2}$, Jaewon Oh ${ }^{3}$, Chan Joo Lee ${ }^{3}$, Sungha Park ${ }^{3}$, \\ Seok-Min Kang ${ }^{3}$, Ichiro Manabe, Soo-jin Ann ${ }^{5, *}$ E Sang-Hak Lee ${ }^{3, *}$ \\ ${ }^{1}$ Graduate Program of Science for Aging, Yonsei University, Seoul 03722, ${ }^{2}$ Department of Biostatistics and Computing, Graduate School of \\ Yonsei University, Seoul 03722, ${ }^{3}$ Division of Cardiology, Department of Internal Medicine, Yonsei University College of Medicine, Seoul 03722, \\ Korea, ${ }^{4}$ Department of Disease Biology and Molecular Medicine, Chiba University Graduate School of Medicine, Chiba 260-8670, Japan, \\ ${ }^{5}$ Integrative Research Center for Cerebrovascular and Cardiovascular Diseases, Yonsei University College of Medicine, Seoul 03722, Korea
}

Our understanding of the differential effects between specific omega-3 fatty acids is incomplete. Here, we aimed to evaluate the effects of docosahexaenoic acid (DHA) and eicosapentaenoic acid (EPA) on T-helper type 1 (Th1) cell responses and identify the pathways associated with these responses. Naïve $\mathrm{CD4}^{+} \mathrm{T}$ cells were co-cultured with bone marrow-derived dendritic cells (DCs) in the presence or absence of palmitate (PA), DHA, or EPA. DHA or EPA treatment lowered the number of differentiated IFN- $\gamma$-positive cells and inhibited the secretion of IFN- $\gamma$, whereas only DHA increased IL-2 and reduced TNF- $\alpha$ secretion. There was reduced expression of MHC II on DCs after DHA or EPA treatment. In the DC-independent model, DHA and EPA reduced Th1 cell differentiation and lowered the cell number. DHA and EPA markedly inhibited IFN- $\gamma$ secretion, while only EPA reduced TNF- $\alpha$ secretion. Microarray analysis identified pathways involved in inflammation, immunity, metabolism, and cell proliferation. Moreover, DHA and EPA inhibited Th1 cells through the regulation of diverse pathways and genes, including Igf1 and Cpt1a. Our results showed that DHA and EPA had largely comparable inhibitory effects on Th1 cell differentiation. However, each of the fatty acids also had distinct effects on specific cytokine secretion, particularly according to the presence of DCs. [BMB Reports 2021; 54(5): 278-283]

*Corresponding authors. Sang-Hak Lee, Tel: +82-2-2228-8460; Fax: +82-2-2227-7732; E-mail: shl1106@yuhs.ac; Soo-jin Ann, Tel: +82-22228-8460; Fax: +82-2-2227-7732; E-mail: hoppum@yuhs.ac ${ }^{\#}$ These authors contributed equally to this work.

https://doi.org/10.5483/BMBRep.2021.54.5.267

Received 6 December 2020, Revised 19 January 2021, Accepted 27 April 2021

Keywords: Adaptive immunity, Atherosclerosis, Co-culture technique, Interleukin, Unsaturated fatty acids

\section{INTRODUCTION}

Omega-3 fatty acids, especially eicosapentaenoic acid (EPA), have shown remarkable cardiovascular benefits in a recent clinical trial (1). EPA was observed to reduce cardiovascular risk in all subgroups based on triglyceride levels, suggesting that the noted benefit can be independent of triglyceride reduction (2). However, we only partially understand the mechanisms of action of omega-3 fatty acids, particularly those involved in cardiovascular risk reduction.

Effects of omega-3 fatty acids on lipoprotein metabolism and plaque stabilization, and anti-inflammatory, anti-oxidation, and anti-thrombosis effects are considered to be possible routes of action $(3,4)$. It has been reported that docosahexaenoic acid (DHA) and EPA can affect the functions of macrophages and neutrophils (5). However, studies concerning the cardiovascular effects of DHA or EPA and their mechanisms of action are highly limited, particularly in T cells.

A balance between functional phenotypes of T cells is known to contribute to atherosclerosis progression (6). Immune reaction in atherosclerosis is regarded as pathologic response of $\mathrm{T}$ helper cells with proinflammatory cytokine secretion (7). While a relationship between $\mathrm{T}$ cells, such as $\mathrm{CD} 4^{+} \mathrm{T}$ cells, and coronary events has been reported (8), the effects of T cells on atherosclerosis can vary depending on their subtype, thus limiting clinical applications of T cell-associated targets. Although omega-3 fatty acids are reportedly immunosuppressive in many cell types, including $\mathrm{T}$ cells, much remains unknown regarding their effects in these cells. Particularly, the knowledge of the differential effects between specific omega-3 fatty acids on T-cell response is not comprehensive (9). Therefore, the current study aims to evaluate the effects of DHA and EPA on Th1 cell responses including differentiation, proliferation, and cytokine secretion. Palmitate (PA) was used as a comparison. We measured the effects of DHA and EPA in the presence or absence of dendritic cells (DCs) to investigate whether the differences between fatty acids are influenced by DCs. Finally, we analyzed

ISSN: 1976-670X (electronic edition)

Copyright (C) 2021 by the The Korean Society for Biochemistry and Molecular Biology

(c) This is an open-access article distributed under the terms of the Creative Commons Attribution Non-Commercial License (http://creativecommons.org/licenses/by-nc/4.0) which permits unrestricted non-commercial use, distribution, and reproduction in any medium, provided the original work is properly cited. 
and validated the genes and pathways regulated by the two fatty acids in Th1 cells.

\section{RESULTS}

DHA and EPA, but not PA, inhibited DC-dependent Th1 cell differentiation, proliferation, and cytokines

The purity of CD4 positivity in isolated cells was $>90 \%$ (Supplementary Fig. 1). A DC-dependent Th1 cell differentiation model was used to assess the effects of each fatty acid. Naïve $\mathrm{CD}^{+} \mathrm{T}$ cells were co-cultured with BMDCs for 4 days in the presence or absence of PA, DHA, or EPA ( $50 \mu \mathrm{M}$, respectively). The number of IFN- $\gamma$, a Th1 cell marker, -positive cells was significantly lower after DHA or EPA, but not PA treatment (Fig. 1A, B). Compared to the positive control group, the cell number was reduced upon DHA treatment. The cell number did not significantly change after PA or EPA treatment (Fig. 1C).

In the co-culture model, DHA and EPA markedly inhibited the secretion of IFN- $\gamma$, whereas only DHA significantly reduced that of TNF- $\alpha$ (Fig. 1D). Similarly, DHA and EPA attenuated the expression of Ifng. No considerable alteration was observed in the expression of Tnf (Fig. 1E). PA did not influence the secretion of cytokines or the expression of the corresponding genes (Fig. 1D, E). Only DHA increased IL-2 secretion, while DHA and EPA upregulated the corresponding gene (Fig. 1D, E). EPA significantly decreased the expression of Tbet, a transcription factor for Ifng, whereas the effect of DHA on Tbet expression was not significant (Fig. 1E).

\section{DHA and EPA reduced surface molecules on DCs}

BMDCs were stimulated by lipopolysaccharide (LPS) $(100 \mathrm{ng} / \mathrm{ml})$ with or without PA, DHA, or EPA for 2 days. Representative histograms and mean fluorescence intensities (MFIs) are shown in Fig. 2. The levels of IL-12p40 and IL-12p70 in the media were measured using ELISA. DHA and EPA markedly decreased the expression of major histocompatibility complex (MHC) II, a co-stimulatory molecule expressed on DCs. Neither DHA nor EPA affected the expression of CD11c, CD80, or CD86. PA did not influence the expression of the three molecules (Fig. 2A). IL-12p70 level was reduced after treatment with $\mathrm{DHA}$, whereas this change was not significant with EPA. The levels of IL-12p40 were not altered (Fig. 2B).

\section{DHA and EPA inhibited DC-independent Th1 cell differentiation, proliferation, and cytokines}

To determine bone marrow-derived dendritic cell (BMDC)-independent Th1 cell differentiation, cell culture plates were coated with anti-CD3 $\varepsilon$ and anti-CD28. Naïve $\mathrm{CD}^{+}{ }^{+} \mathrm{T}$ cells were then cultured in these plates for 4 days in the presence or absence of PA, DHA, or EPA $(50 \mu \mathrm{M})$. DHA, EPA, and PA reduced the differentiation of Th1 cells, while the effect of DHA and EPA was more remarkable (Fig. 3A, B). Additionally, DHA and EPA inhibited the proliferation of Th1 cells (Fig. 3C).

$\mathrm{DHA}, \mathrm{EPA}$, and PA decreased IFN- $\gamma$ secretion, whereas the
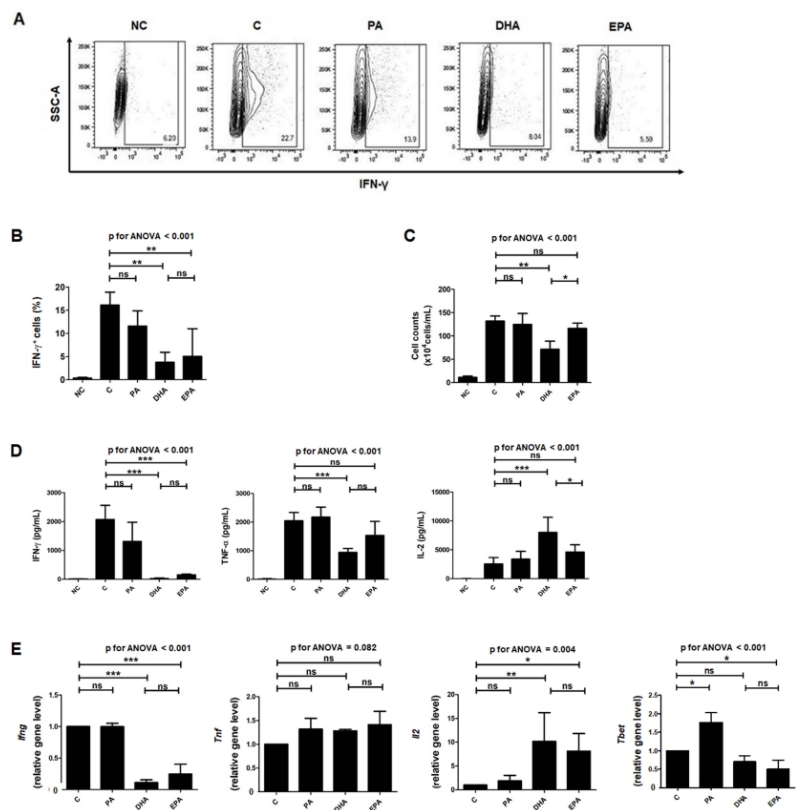

Fig. 1. Effects of palmitate (PA), docosahexaenoic acid (DHA), and eicosapentaenoic acid (EPA) on differentiation, proliferation, and cytokine secretion of T-helper type 1 (Th1) cells in a co-culture model. Naïve $\mathrm{CD}^{+}{ }^{+} \mathrm{T}$ cells were co-cultured in the presence or absence of PA, DHA, or EPA $(50 \mu \mathrm{M})$. Intracellular IFN- $\gamma$ production among differentiated $\mathrm{CD}^{+} \mathrm{T}$ cells was analyzed by FACS analysis. Representative FACS plots (A) and relative quantification (B) indicate the effects of each fatty acid on differentiation of Th1 cells. Cell number was determined using a hemocytometer $(\mathrm{C})$. In the same co-culture model with or without PA, DHA, or EPA, cytokine levels were determined by ELISA assay (D). Relative mRNA expression of Ifng, Tnf, 112, and Tbx21 was measured by real-time PCR after cell differentiation. Expression levels were normalized to mRNA levels of $\beta$-actin (E). ELISA and qPCR were conducted with technical duplicates, and data were obtained from at least three independent experiments. ${ }^{*} \mathrm{P}<0.05 ;{ }^{*} \mathrm{P}<0.01$; ${ }^{* *} \mathrm{P}<0.001$; ns: not significant compared with the control group; NC: negative control; C: control; PA: palmitic acid.

effect of DHA or EPA was more obvious (Fig. 3D). Conversely, only EPA dramatically reduced the secretion of TNF- $\alpha$. DHA and EPA remarkably inhibited Ifng expression, whereas the effects of DHA or EPA on Tnf were insignificant (Fig. 3E). The effects of the three fatty acids on IL-2 secretion and the expression of the corresponding gene were not significant (Fig. 3D, E). The expression of Thet was reduced by PA, DHA, and EPA (Fig. 3E).

\section{Effects of PA on Th1 cell responses}

The effect of PA was not significant on the differentiation, proliferation, and cytokine secretion of Th1 cells in the co-culture model. PA had a largely similar effect in a DC-independent model, but reduced cell differentiation and IFN- $\gamma$ secretion from Th1 cells. 

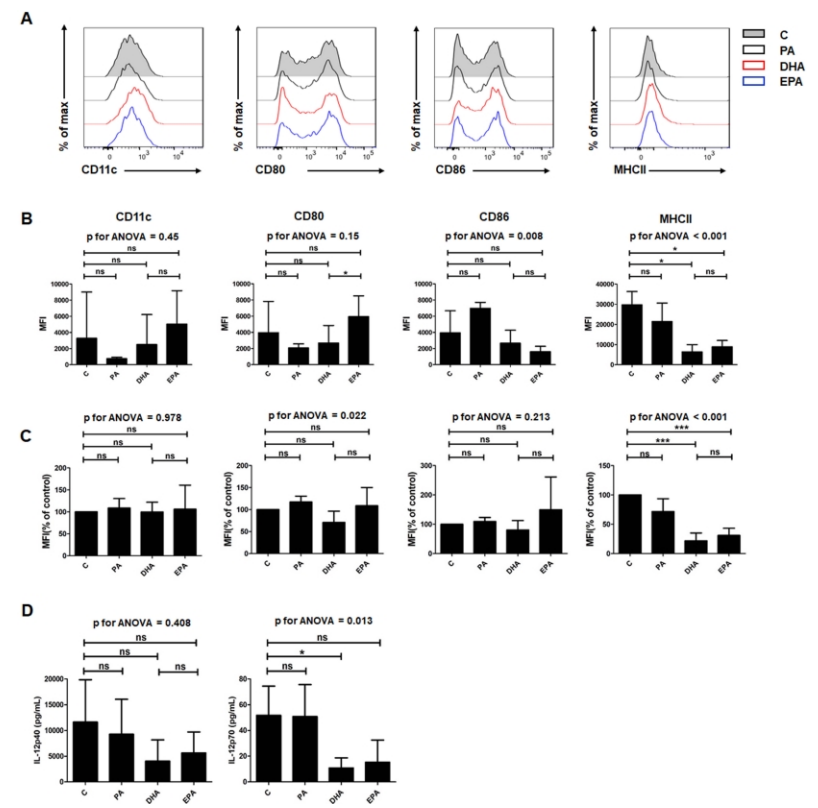

Fig. 2. Effects of PA, DHA, and EPA on surface markers of BMDCs and IL-12p70 production. BMDCs were stimulated by LPS with or without PA, HAD, or EPA for 2 days. Representative FACS plots (A) and mean fluorescence intensity (MFI) (B) of CD11C, CD80, and $\mathrm{MHCll}$ expression are shown. MFI values for each group of cells were normalized to control (C). The levels of IL-12p40 and IL-12p70 in the culture media were measured by ELISA (D). Data were obtained from at least three independent experiments. $* P<$ $0.05 ; * * \mathrm{P}<0.01 ; * * * \mathrm{P}<0.001$; ns: not significant compared with the control group; NC: negative control; C: control; PA: palmitic acid.

Genes and pathways of Th1 cells regulated by DHA and EPA To identify the genes of Th1 cells altered by DHA, naïve CD4 ${ }^{+}$ $T$ cells were cultured with BMDCs for 4 days with or without $\mathrm{DHA}(50 \mu \mathrm{M})$. Using a microarray analysis, DEGs were compared after treatment with vehicle or DHA (Supplementary Fig. 1A). To determine the biologically relevant genes affected by DHA, we selected genes in the top ten pathways ranked by the analysis (Supplementary Table 1).

The associated pathways included but were not limited to, cytokine-cytokine receptor interaction, chemokine signaling, and fatty acid metabolism. Among the pathways, we focused on the Ras signaling pathway. Genes, including Tiam1, Efna5, and Igf1 were upregulated, whereas those including Prkacb, Gng2, Grin1, and Vegfa were downregulated by DHA (Supplementary Table 1). Quantitative polymerase chain reaction (qPCR) for validation showed that expression levels of Igf1 significantly increased, whereas changes in other genes were not significant (Supplementary Fig. 1B).

To identify genes of Th1 cells affected by EPA, the same co-culture model, microarray analysis, and comparisons were used. The associated pathways included but were not limited to those involved in regulation of the hematopoietic cell lineage,
A
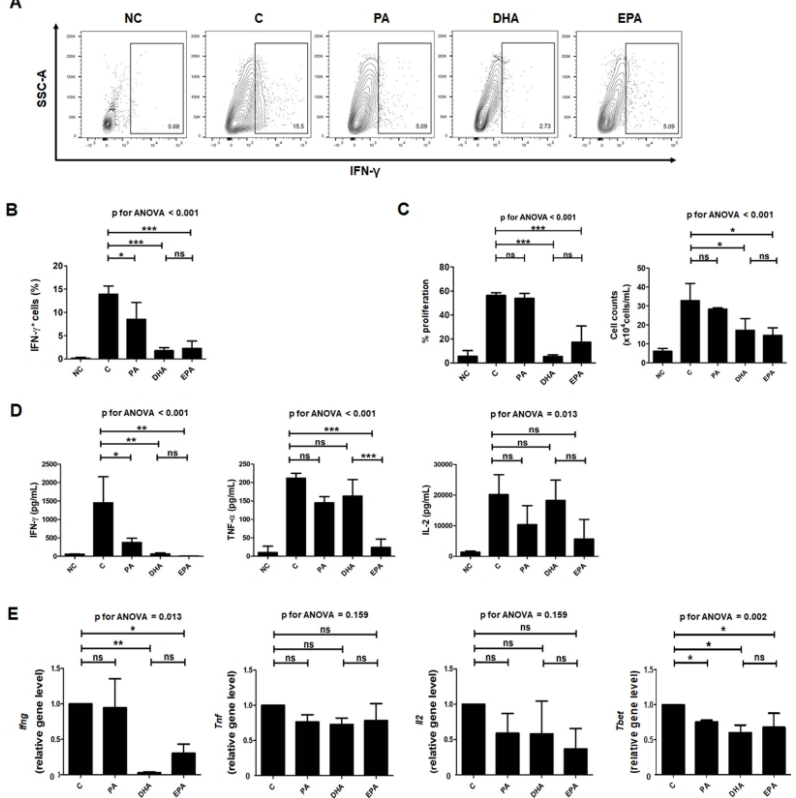

Fig. 3. Effects of PA, DHA and EPA on BMDC-independent differentiation, proliferation, and cytokine secretion of Th1 cells. BMDCindependent Th1 cell differentiation was analyzed using cell culture in plates pre-coated with anti-CD3 $\varepsilon$ and anti-CD28. Naïve CD4 ${ }^{+} \mathrm{T}$ cells were then cultured in the plates in the presence or absence of PA, DHA, or EPA. Cell-surface expression of IFN- $\gamma$ was analyzed by flow cytometry (A and B). The effects of DHA and EPA on Th1 cell proliferation and cell number were determined by CFSE assay and flow cytometry $(\mathrm{C})$. The cytokine secretion and corresponding relative mRNA levels of IFN- $\gamma$, TNF- $\alpha$, and IL-2 were determined (D and E). mRNA levels of T-box transcription factor were measured by real-time PCR and are expressed as a ratio to GAPDH. The results of real-time PCR are expressed as a ratio to $\beta$-actin. Both ELISA and QPCR were conducted in technical duplicates, and data were obtained from at least three independent experiments. ${ }^{*} \mathrm{P}<0.05$; $* * \mathrm{P}<0.001$; and $* * * \mathrm{P}<0.0001$ compared with the control group. NC: negative control; C: control; ns: not significant.

cytokine-cytokine receptor interaction, and peroxisome proliferator-activated receptor signaling. Genes including Cpt1a and Slc27a2 were upregulated, whereas several others, including Runx2, Vegfa, and Itga2, were downregulated by EPA (Supplementary Table 1 and Supplementary Fig. 1D). The upregulation of Cpt1a and downregulation of Runx2 and Itga2 were validated (Supplementary Fig. 1E).

Gene ontology (GO) categories for DEGs by DHA included immune system process and response, positive regulation of inflammation, and cytokine activity. Those of DEGs by EPA included signal transduction, immune response, defense response to protozoa, positive regulation of $\mathrm{T}$ cells, and chemokine receptor activity (Supplementary Fig. 1C, F). Further experiments for validation of these role were conducted by inhibiting the Igf1 gene and Cpt-1a. Inhibition of Cpt-1a by etomoxir partly, but significantly, restored differentiation of Th1 cells decreased 
by EPA. Although silencing of Igf1 by siRNA modestly increased differentiation, the difference was not statistically significant (Supplementary Fig. 1G).

\section{DISCUSSION}

The major findings of this study were as follows: DHA and EPA inhibited the differentiation of Th1 cells, regardless of the presence of DCs; DHA and EPA inhibited the DC-dependent differentiation of INF- $\gamma$ positive cells and the secretion of IFN- $\gamma$, whereas only DHA increased IL-2 reduced TNF- $\alpha$ secretion; DC-dependent effects of DHA and EPA were mediated, at least in part, by the downregulation of MHC II; the inhibitory effects on cytokines were more prominent for $\mathrm{DHA}$ in the presence of DCs and for EPA in the absence of DCs; PA did not have similar effects on Th1 cells. The working pathways of DHA and EPA in Th1 cells included but were not limited to those of inflammation, immunity, metabolism, and cell proliferation. The regulation of several genes, including Igf1 and Cpt1a, was also validated in this study. Notably, we demonstrated, for the first time, the distinct effects of DHA and EPA on specific cytokine secretion according to the presence of DCs, although these two fatty acids had largely similar inhibitory effects on Th1 cells.

A recent study showed that EPA and DHA have similar antiinflammatory effects on $\mathrm{CD} 4^{+} \mathrm{T}$ cells as assessed using migration assays (10). The relatively neutral effect of PA on these T cells is in concordance with our findings. In our study, although EPA and DHA showed similar effects in Th1 cells, the effects of DHA tended to be more prominent in the presence of DCs, whereas some effects of EPA were more obvious in the DC-independent model. Currently, it is not fully understood how these two fatty acids exert distinct effects on cytokines. Possibly, their different inhibitory effects on DC, for example on surface molecules unexamined in our study (11), could be associated. Conversely, mammalian target of rapamycin (mTOR) on $\mathrm{T}$ cell is a crucial molecule for cellular metabolism and $\mathrm{T}$ cell differentiation and function (12). Furthermore, omega-3 fatty acids are known to downregulate mTOR (13). Therefore, the effect of individual omega- 3 fatty acids on T cell surface molecules, including mTOR, can differ, and this could account for the differences in cytokine secretion, as in our study.

In the current study, the inhibitory effects of EPA on DCindependent TNF- $\alpha$ secretion were more obvious than those of the other fatty acids. TNF- $\alpha$ is reportedly associated with a high incidence of cardiovascular events (14). Reduction of TNF- $\alpha$ secretion in Th1 cells by EPA may be one of many biological mechanisms inducing clinical benefits. As shown in this study, DHA and EPA had differential effects on some responses of Th1 cells. Previously reported anti-inflammatory effects of DHA and EPA on diverse tissues and pathological environments related to TNF- $\alpha(15,16)$. Accordingly, our results demonstrating the distinct effects of DHA and EPA may provide insights into the mechanisms underlying their benefits in differ- ent contexts rather than superiority of one over another.

A prior study revealed that DHA and EPA directly influenced and attenuated the secretion of IL-2 and the proliferation of T cells (17). Conversely, T cells of mice on a DHA- or EPA-enriched diet did not show a reduction in IL-2 transcription, while they suppressed IL-2R mRNA levels (18). We found that elevated IL-2 secretion was more dramatic following DHA treatment in the DC-dependent model. This might be one example of differential effects of omega- 3 fatty acids according to the presence of DCs. In addition, it was of note that the increased IL-2 in the DC-dependent model was not accompanied by an enhanced T-cell proliferation. Recent studies identified the effects of IL-2 on regulatory T-cell expansion and protection from atherosclerosis (19). It would thus be of interest to evaluate whether IL-2 elevation in the current study is linked to the omega-3 fatty acid-driven cardiovascular benefit observed in recent clinical studies (1).

DHA or EPA treatment markedly reduced the expression of MHC II molecules. This could be one of the pivotal links between the two fatty acids and their DC-dependent effect. This finding was in line with other studies (20). In the current study, the effects of DHA and EPA on CD80 or CD86 were less clear than those on MHC II. DHA and EPA are precursors of specialized pro-resolving lipid mediators $(9,21)$ that can affect T-cell differentiation through regulating transcription factors (22). Among them, the resolvin D1 analog suppresses MHC II and CD40 expression, whereas it did not affect CD80 and CD86 expression (23). Accordingly, pro-resolving mediators, such as resolvin D1, seem to modulate the interactions between DCs and T cells, preferably through the CD40/154 pathway rather than the CD80/86-CD28 pathway. This is in concordance with our observation. However, we did not examine the effects of lipid mediators but only of omega-3 fatty acids themselves. Thus, we cannot rule out the potential pathways in DCs, independent of mediators such as resolvins.

Studies performed in the past decades discovered that DHA and EPA change the basic properties of cell membranes, modulate lipid microdomains and immunological synapses, and regulate downstream cell signaling cascades and nuclear receptor activation (24). It was also suggested that the effects of DHA on T cells could be related to altered microdomains and downstream signaling $(25,26)$. In a model membrane, DHA influenced the membrane structure and fluidity, whereas EPA accumulated cholesterol-rich domains and inhibited inflammation (27). Although the association between the differential effects of the two fatty acids and changes in the membrane is beyond the scope of our study, it will be of interest to explore the issues in future studies.

The genes upregulated by DHA included Igf1. Observations of the effects of insulin-like growth factor signaling on atherosclerosis have been conflicting (28). The role of this gene has been evaluated in various cell types, including smooth muscle cells (29) and macrophages (30). Recombinant human insulinlike growth factor-1 (rhlGF-1) delivery has been shown to 
increase the number of T cells in mice (31). Although rhIGF-1 stimulated the proliferation of regulatory $\mathrm{T}$ cells, it did not affect the proliferation of other proinflammatory $T$ cell subsets (32). Conversely, a recent study reported that knockout of macrophage-derived IGF-1 gene in mice elevated Th1 (IFN- $\left.\gamma^{+}\right)$ cell proportions (33). This indicates that IGF-1 or Igf1 may be involved in the inhibition of Th1 cell differentiation. Nevertheless, because research results vary, whether Igf1 plays a primary role in the proliferation and differentiation of Th1 cells needs to be further elucidated. Particularly, silencing of Igf1 did not show dramatic impact on differentiation of Th1 cells post $\mathrm{DHA}$ treatment, and the mechanism of these effects remains to be additionally elucidated.

The genes validated in our study include Cpt1a, a target gene of PPAR $\alpha$, which encodes carnitine palmitoyltransferase I (Cpt1). We demonstrated that upregulation of Cpt1a contributes, at least in part, to the inhibitory effect of EPA on Th1 cell differentiation. CPT1 contributes to mitochondrial fatty acid oxidation. It has been reported that EPA induces the expression of CPT1A in intestinal cells (34). In a mouse study, knocking out Cpt1a in endothelial cells promoted leukocyte infiltration, implying that Cpt1a may be atheroprotective (35). Furthermore, mitochondrial fatty acid oxidation, for which CPT1A has an important role, is reported to be essential for the survival and activation of $\mathrm{CD}^{+} \mathrm{T}$ cells (36). Interestingly, PD-1, a $T$ cell surface protein, has been reported to promote fatty acid oxidation by increasing CPT1A expression. Because $\mathrm{T}$ cells utilize glycolysis during differentiation to effectors, this is responsible for PD-1-induced inhibition of $\mathrm{T}$ cell differentiation (37). In addition, this is in line with our finding that Cpt1a upregulation concomitantly suppressed T cell responses. Collectively, it is possible that Cpt1a plays a role in multiple T-cell subtypes contributing to the vascular condition.

Our study has potential limitations. We investigated the response of Th1 cells in DC-dependent or -independent manners. However, because there are other T-cell types involved in vascular immune response, our results may not provide a completely integrated perspective. Nevertheless, the primary purpose of our study was to evaluate the effects of DHA and EPA on Th1 cell responses. Additionally, we designed this study to perform only in vitro experiments. This nature of our current study has limitations when attempting to extrapolate the results to animal models or the clinical setting. However, we performed a comprehensive analysis to compare cellular responses induced by each of the target fatty acids.

DHA and EPA inhibited the activation of Th1 cells in both DC-dependent and -independent manners. Modest but significant differential effects between DHA and EPA on Th1 cells were demonstrated for the first time. These two fatty acids regulated diverse genes, including Igf1 and Cpt1a in Th1 cells. Further studies of these differential effects on adaptive immunity are needed to clarify their beneficial effects on diverse sites and pathological conditions, including cardiovascular diseases.

\section{MATERIALS AND METHODS}

Detailed methods are described in Supplementary Material.

\section{ACKNOWLEDGEMENTS}

This work was supported by the National Research Foundation of Korea grant funded by the Korean government (grant numbers: 20181D1A1B07043855 and 2019R1F1A1057952). The funder had no role in the design and conduct of the study; collection, management, analysis, and interpretation of the data; preparation, review, or approval of the manuscript; and decision to submit the manuscript for publication.

\section{CONFLICTS OF INTEREST}

The authors have no conflicting interests.

\section{REFERENCES}

1. Bhatt DL, Steg PG, Miller M et al (2019) Cardiovascular risk reduction with icosapent ethyl for hypertriglyceridemia. N Engl J Med 380, 11-22

2. Bhatt DL, Steg PG, Miller M et al (2019) Reduction in first and total ischemic events with icosapent ethyl across baseline triglyceride tertiles. J Am Coll Cardiol 74, 1159-1161

3. Kawashiri MA, Tada H, Yamagishi M (2018) Unsolved antiatherogenic mechanism of $n-3$ polyunsaturated fatty acids. Circ J 82, 332-333

4. Son JW, Kim CH, Nam MS, Park IB, Yoo SJ (2019) Efficacy and safety of prescription of omega-3 fatty acids added to stable statin therapy in Korean patients with type 2 diabetes and hypertriglyceridemia: a randomized controlled trial. J Lipid Atheroscler 8, 221-231

5. Gutiérrez S, Svahn SL, Johansson ME (2019) Effects of omega-3 fatty acids on immune cells. Int J Mol Sci 20, 2476

6. Tabas I and Lichtman AH (2017) Monocyte-macrophages and $T$ cells in atherosclerosis. Immunity 47, 621-634

7. Winkels H, Wolf D (2020) Heterogeneity of T cells in atherosclerosis defined by single-cell RNA-sequencing and cytometry by time of flight. Arterioscler Thromb Vasc Biol 41, 549-563

8. Tomas L, Bengtsson E, Andersson L et al (2020) Low levels of $\mathrm{CD}^{+}{ }^{+} \mathrm{CD} 28{ }^{\text {null }} \mathrm{T}$ cells at baseline are associated with first-time coronary events in a prospective population-based case-control cohort. Arterioscler Thromb Vasc Biol 40, 426-436

9. Duffney PF, Falsetta ML, Rackow AR, Thatcher $T H$, Phipps RP, Sime PJ (2018) Key roles for lipid mediators in the adaptive immune response. J Clin Invest 128, 2724 2731

10. Cucchi D, Camacho-Muñoz D, Certo M et al (2020) Omega-3 polyunsaturated fatty acids impinge on CD4+ T cell motility and adipose tissue distribution via direct and lipid mediator-dependent effects. Cardiovasc Res 116, 1006-1020 
11. Kong W, Yen JH, Vassiliou E, Adhikary S, Toscano MG, Ganea D (2010) Docosahexaenoic acid prevents dendritic cell maturation and in vitro and in vivo expression of the IL-12 cytokine family. Lipids Health Dis 9, 12

12. Waickman AT, Powell JD (2012) mTOR, metabolism, and the regulation of T-cell differentiation and function. Immunol Rev 249,43-58

13. Chen Z, Zhang Y, Jia $C$ et al (2014) mTORC1/2 targeted by $n-3$ polyunsaturated fatty acids in the prevention of mammary tumorigenesis and tumor progression. Oncogene 33, 4548-4557

14. Ridker PM, Rifai N, Pfeffer M, Sacks F, Lepage S, Braunwald E (2000) Elevation of tumor necrosis factor-alpha and increased risk of recurrent coronary events after myocardial infarction. Circulation 101, 2149-2153

15. Yamagata K, Suzuki S, Tagami M (2016) Docosahexaenoic acid prevented tumor necrosis factor alpha-induced endothelial dysfunction and senescence. Prostaglandins Leukot. Essent Fatty Acids 104, 11-18

16. Albracht-Schulte K, Gonzalez S, Jackson A et al (2019) Eicosapentaenoic acid improves hepatic metabolism and reduces inflammation independent of obesity in high-fat-fed mice and in HepG2 cells. Nutrients 11, 599.

17. McMurray DN, Jolly CA, Chapkin RS (2000) Effects of dietary $n-3$ fatty acids on $T$ cell activation and $T$ cell receptor-mediated signaling in a murine model. J Infect Dis 182 Suppl 1, S103-107

18. Jolly CA, McMurray DN, Chapkin RS (1998) Effect of dietary n-3 fatty acids on interleukin-2 and interleukin-2 receptor alpha expression in activated murine lymphocytes. Prostaglandins Leukot. Essent Fatty Acids 58, 289-293

19. Dietrich T, Hucko T, Schneemann C et al (2012) Local delivery of IL-2 reduces atherosclerosis via expansion of regulatory T cells. Atherosclerosis 220, 329-336

20. Hoppenbrouwers T, Cvejić Hogervorst JH, Garssen J, Wichers HJ, Willemsen LEM (2019) Long chain polyunsaturated fatty acids (LCPUFAs) in the prevention of food allergy. Front Immunol 10, 1118

21. Serhan CN (2014) Pro-resolving lipid mediators are leads for resolution physiology. Nature 510, 92-101

22. Chiurchiù V, Leuti A, Dalli J et al (2016) Proresolving lipid mediators resolvin D1, resolvin D2, and maresin 1 are critical in modulating T cell responses. Sci Transl Med 8, 353ra111

23. Hua J, Jin Y, Chen Y et al (2014) The resolvin D1 analogue controls maturation of dendritic cells and suppresses alloimmunity in corneal transplantation. Invest Ophthalmol Vis Sci 55, 5944-5951

24. Kim W, Khan NA, McMurray DN, Prior IA, Wang N, Chapkin RS (2010) Regulatory activity of polyunsaturated fatty acids in T-cell signaling. Prog Lipid Res 49, 250-261
25. Fan YY, Fuentes NR, Hou TY et al (2018) Remodelling of primary human $\mathrm{CD} 4+\mathrm{T}$ cell plasma membrane order by n-3 PUFA. Br J Nutr 119, 163-175

26. Fan YY, Ly LH, Barhoumi R, McMurray DN, Chapkin RS (2004) Dietary docosahexaenoic acid suppresses T cell protein kinase $\mathrm{C}$ theta lipid raft recruitment and IL-2 production. J Immunol 173, 6151-6160

27. Mason RP, Jacob RF, Shrivastava S, Sherratt SCR, Chattopadhyay A (2016) Eicosapentaenoic acid reduces membrane fluidity, inhibits cholesterol domain formation, and normalizes bilayer width in atherosclerotic-like model membranes. Biochim Biophys Acta 1858, 3131-3140

28. Yu XH, He LH, Gao JH, Zhang DW, Zheng XL, Tang CK (2018) Pregnancy-associated plasma protein-A in atherosclerosis: molecular marker, mechanistic insight, and therapeutic target. Atherosclerosis 278, 250-258

29. Sukhanov S, Higashi Y, Shai SY et al (2018) SM22 $\alpha$ (smooth muscle protein 22- $\alpha$ ) promoter-driven IGF1R (insulin-like growth factor 1 receptor) deficiency promotes atherosclerosis. Arterioscler Thromb Vasc Biol 38, 2306-2317

30. Higashi Y, Sukhanov S, Shai SY et al (2016) Insulin-like growth factor-1 receptor deficiency in macrophages accelerates atherosclerosis and induces an unstable plaque phenotype in apolipoprotein E-deficient mice. Circulation 133, 2263-2278

31. Clark R, Strasser J, McCabe S, Robbins K, Jardieu P (1993) Insulin-like growth factor-1 stimulation of lymphopoiesis. J Clin Invest 92, 540-548

32. Bilbao D, Luciani L, Johannesson B, Piszczek A, Rosenthal N (2014) Insulin-like growth factor-1 stimulates regulatory $\mathrm{T}$ cells and suppresses autoimmune disease. EMBO Mol Med 6, 1423-1435

33. Yoon IS, Park H, Kwak HW, Jung YW, Nam JH (2017) Macrophage-derived insulin-like growth factor-1 affects influenza vaccine efficacy through the regulation of immune cell homeostasis. Vaccine 35, 4687-4694

34. Mashurabad PC, Kondaiah P, Palika R, Ghosh S, Nair MK, Raghu P (2016) Eicosapentaenoic acid inhibits intestinal $\beta$-carotene absorption by downregulation of lipid transporter expression via PPAR- $\alpha$ dependent mechanism. Arch Biochem Biophys 590, 118-124

35. Nomura M, Liu J, Yu ZX et al (2019) Macrophage fatty acid oxidation inhibits atherosclerosis progression. J Mol Cell Cardiol 127, 270-276

36. Raud B, McGuire PJ, Jones RG, Sparwasser T, Berod L (2018) Fatty acid metabolism in CD8 + T cell memory: challenging current concepts. Immunol Rev 283, 213-231

37. Patsoukis N, Bardhan K, Chatterjee P et al (2015) PD-1 alters T-cell metabolic reprogramming by inhibiting glycolysis and promoting lipolysis and fatty acid oxidation. Nat Commun 6, 6692 